\title{
Comparative Study of Dynamic Phasor and Harmonic State-Space Modeling for Small-Signal Stability Analysis
}

\author{
Philippe De Rua, Özgür Can Sakinci, Jef Beerten \\ KU Leuven ESAT/ELECTA Research Group \& EnergyVille \\ Leuven \& Genk, Belgium \\ \{philippe.derua, ozgurcan.sakinci, jef.beerten\}@esat.kuleuven.be
}

Please cite this article as:

P. De Rua, O. C. Sakinci, and J. Beerten, "Comparative Study of Dynamic Phasor and Harmonic State-Space Modeling for Small-Signal Stability Analysis," Electric Power Systems Research, vol. 189, no. 106626, 2020.

\begin{abstract}
This paper conducts a comparison of two promising frequency-lifted representations used in the state-space modeling of power-electronic converters: dynamic phasors and harmonic state-space. These methods originate from similar hypotheses and aim to tackle the time-periodicity problem observed in systems with multiple harmonic components in steady state. The paper derives the theoretical foundations of the methods with an emphasis on their similarities and differences, and applies them to a two-level voltage-source converter. The application demonstrates the effects of different truncation orders of the models infinite formulations. Stability analysis tools such as eigenvalues and transfer functions are examined and benchmarked against a classical dq-frame small-signal model.
\end{abstract}

Index Terms-dynamic phasor, harmonic state-space, linear time-periodic systems, small-signal modeling, voltage-source converters.

\section{INTRODUCTION}

The increasing use of power-electronic converters and the phase-out of synchronous-generator-based power plants raise power quality and system stability concerns. In particular, new types of control interactions with the network and between converters are becoming more frequent as a consequence of enlarged control bandwidths and decreased electrical damping [1]. To study the small-signal dynamics of such systems, there is a growing interest in capturing the impact of converter controls and modulation on the coupling of different harmonic components as accurately as possible. This is a challenging task, as the mathematical models are nonlinear and timeperiodic in steady state. Park's transformation has been used extensively to tackle the problem of time-periodicity, but it presents limitations, particularly when studying single-phase

This work is part of the Neptune project, supported by the Energy Transition Fund, FOD Economy, Belgium. The work of P. De Rua and J. Beerten has been supported by the Research Foundation - Flanders (FWO) (Grant No. $11 \mathrm{E} 8720 \mathrm{~N}$ and $12 \mathrm{D} 1117 \mathrm{~N})$
AC-DC systems, unbalanced three-phase AC-DC systems and, more generally, systems involving multi-frequency signals. Such studies require the application of relatively new sophisticated modeling methods [1], [2]. Examples of such methods are the dynamic phasors (DPs) framework and the harmonic state-space (HSS) modeling method, which provide equivalent time-invariant representations of multi-frequency time-periodic systems, overcoming Park's transformation limitations while preserving the possibility of applying classical stability theories for time-invariant systems.

The DP framework was introduced by Sanders et al., who presented an averaged modeling method for power-electronic circuits [3]. This framework mainly focused on reducing the simulation time of such circuits, as the band-limited DPs made it possible to use larger simulation time steps [4]. More recently, DPs have also been used for small-signal stability analysis based on eigenvalues [5]-[8].

The HSS framework was first introduced by Wereley [9] for the stability analysis of mechanical applications. The method has also been discussed under different names such as the Extended Harmonic Domain [10], [11]. Since, it has been used to study various systems, including the single-phase two-level converter [12] and the modular multilevel converter [13].

In [14], the foundations of several harmonic modeling methods are reviewed, and the focus is set on proving their mathematical equivalence. In particular, DPs and HSS modeling methods originate from similar initial hypotheses and they are both based on the technique of frequency lifting. This technique consists in representing signals by a set of timevarying Fourier coefficients in a higher-dimensional space to obtain time-invariant representations of time-periodic systems [15]. Both methods are now extensively used for stability analysis. However, to the authors' knowledge, a comparison of real-valued DPs with HSS showing the impact of modeling choices on the stability assessment is yet to be provided. Therefore, this paper thoroughly compares these two promising modeling methods, and provides insights into their mathematical underpinnings before applying them to a twolevel voltage-source converter (VSC). The comparative study investigates the respective advantages and limitations of DPs and HSS for the stability analysis of time-periodic systems using linear systems tools such as eigenvalues and transfer 
functions.

The paper is organised as follows. Section II describes the hypotheses of the two modeling methods, presents their analytical formulations and highlights similarities and differences between them. The topology of the VSC and its controller are introduced in Section III. In Section IV, the modeling methods are applied to the equations of the VSC. In Section V, the comparison is continued with the analysis of eigenvalues and transfer functions, benchmarked against the results of an equivalent $d q$-frame small-signal model. A discussion of advantages and shortcomings of the methods is provided in Section VI, and Section VII concludes the paper.

\section{FREQUENCY-LIFTED MODELING THEORIES}

In what follows, boldface characters refer to vector or matrix quantities. Additionally, the term phasor refers to the complex number that contains phase and magnitude information of a complex sinusoidal quantity.

The dynamic systems considered in this paper are timecontinuous and can be initially expressed as a set of differential equations of the form:

$$
\begin{aligned}
\frac{d \boldsymbol{x}(t)}{d t} & =\boldsymbol{f}(\boldsymbol{x}(t), \boldsymbol{u}(t)), \\
\boldsymbol{y}(t) & =\boldsymbol{g}(\boldsymbol{x}(t), \boldsymbol{u}(t)),
\end{aligned}
$$

where inputs $\boldsymbol{u}$, states $\boldsymbol{x}$, and outputs $\boldsymbol{y}$ are periodic of pe$\operatorname{riod} T_{g}=2 \pi / \omega_{g}$ in steady state, and where functions $\boldsymbol{f}$ and $\boldsymbol{g}$ may be nonlinear but must be continuously differentiable with respect to the elements of $\boldsymbol{x}$ and $\boldsymbol{u}$ on the domain of values taken by these variables. The reason for this requirement is that DPs and HSS models rely on linear approximations of the nonlinear systems. Consequently, $\boldsymbol{f}$ and $\boldsymbol{g}$ may technically comprise products, divisions, rational powers, trigonometric functions and pure time delays, among others; whereas logical operations, hysteresis, dead bands, saturation and piecewise linear functions, among others, cannot be included as such.

\section{A. Dynamic phasors}

The transformation of the initial system (1) into an equivalent linear time-invariant (LTI) formulation based on DPs relies on three aspects: 1) a modification of the signal representation, 2) harmonic balance, and 3) linearization.

1) Signal representation: The theory of DPs originates from the hypothesis that $x(t)$ can be modeled as a nearlyperiodic signal during transients [3]. Consequently, the signal can be written as a Fourier series with time-varying complexvalued Fourier coefficients:

$$
\boldsymbol{x}(t)=\sum_{k=-\infty}^{+\infty} \boldsymbol{X}_{k}(t) e^{j k \omega_{g} t} .
$$

These Fourier coefficients are called dynamic phasors and are denoted by $\langle\boldsymbol{x}\rangle_{k}(t)$. During transients, the DPs are obtained by applying the following moving averaging operator:

$$
\boldsymbol{X}_{k}(t)=\langle\boldsymbol{x}\rangle_{k}(t)=\frac{1}{T_{g}} \int_{t-T_{g}}^{t} \boldsymbol{x}(\tau) e^{-j k \omega_{g} \tau} d \tau
$$

When dynamic variables in (1) are replaced by their Fourier series representations, products translate into convolutions while other continuously-differentiable nonlinear operations such as trigonometric functions or rational powers of variables must be handled using different techniques, which are not discussed here for conciseness.

2) Harmonic balance: Next, it is imposed that samefrequency phasors must be equal. This operation is referred to as harmonic balance [16] and leads to a set of nonlinear differential equations per harmonic index. The initial statespace system is now formulated in the harmonic domain.

Equivalently ${ }^{1}$, the generic index- $k$ set of DP equations can be directly derived from (1) by replacing the states $x(t)$, inputs $u(t)$ and outputs $y(t)$ by their index- $k$ DPs $\langle x\rangle_{k},\langle u\rangle_{k}$ and $\langle y\rangle_{k}$. Time derivatives and multiplications ${ }^{2}$ of signals are then represented in terms of DPs as:

$$
\begin{aligned}
\left\langle\frac{d \boldsymbol{x}}{d t}\right\rangle_{k} & =\frac{d\langle\boldsymbol{x}\rangle_{k}}{d t}+j k \omega_{g}\langle\boldsymbol{x}\rangle_{k}, \\
\left\langle x_{1} x_{2}\right\rangle_{k} & =\sum_{l=-\infty}^{+\infty}\left\langle x_{1}\right\rangle_{l}\left\langle x_{2}\right\rangle_{k-l} .
\end{aligned}
$$

These steps result in a model with complex variables. To obtain a real-valued representation, the real and imaginary parts of the DPs are represented by distinct real-valued variables, e.g. for the states:

$$
\langle x\rangle_{k}=\langle x\rangle_{k}^{R}+j\langle x\rangle_{k}^{I}, \quad\langle x\rangle_{-k}=\langle x\rangle_{k}^{*}=\langle x\rangle_{k}^{R}-j\langle x\rangle_{k}^{I} .
$$

Thus, except for index-0 DPs, a single DP is represented by two variables. Separating real and imaginary parts of the dynamic equations results in a nonlinear real-valued DP model.

3) Linearization: In steady state, all states and inputs are periodic, which implies that all DPs are constant. Thus, a statespace model whose variables are DPs inherently presents a time-invariant operating point in steady state. The equations are linearized around this operating point to obtain an LTI state-space formulation:

$$
\begin{aligned}
\frac{d \boldsymbol{x}_{D P}(t)}{d t} & =\boldsymbol{A} \boldsymbol{x}_{D P}(t)+\boldsymbol{B} \boldsymbol{u}_{D P}(t), \\
\boldsymbol{y}_{D P}(t) & =\boldsymbol{C} \boldsymbol{x}_{D P}(t)+\boldsymbol{D} \boldsymbol{u}_{D P}(t),
\end{aligned}
$$

with $\quad \boldsymbol{x}_{D P}(t)=\left[\left\langle\boldsymbol{x}^{T}\right\rangle_{0}^{R}\left\langle\boldsymbol{x}^{T}\right\rangle_{1}^{R}\left\langle\boldsymbol{x}^{T}\right\rangle_{1}^{I}\left\langle\boldsymbol{x}^{T}\right\rangle_{2}^{R} \cdots\right]^{T}$, where ${ }^{T}$ refers to transposition. Vectors $\boldsymbol{u}_{D P}$ and $\boldsymbol{y}_{D P}$ are defined in a similar way.

The transfer functions of the system can be calculated by applying:

$$
\boldsymbol{Y}(s)=\left(\boldsymbol{C}(s \boldsymbol{I}-\boldsymbol{A})^{-1} \boldsymbol{B}+\boldsymbol{D}\right) \boldsymbol{U}(s),
$$

where $\boldsymbol{I}$ is the identity matrix of appropriate size.

\footnotetext{
${ }^{1}$ For systems that do not present nonlinear operations other than the multiplication of signals

${ }^{2}$ Equation (4b) is defined for scalar DPs rather than vectors, to account for products between scalar elements of $\boldsymbol{x}$ and/or $\boldsymbol{u}$ in $\boldsymbol{f}$ and $\boldsymbol{g}$.
} 


\section{B. Harmonic state space}

Similarly to DPs, the transformation of the initial system (1) into an equivalent LTI formulation based on HSS relies on a modification of signal representation, harmonic balance and linearization, although in a different order.

1) Linearization: Unlike the derivation of the DPs formulation, in which the nonlinear system is first made time invariant and then is linearized around the constant steady-state operating point, the derivation of HSS generally starts from the linearization of the differential equations around a steady-state periodic trajectory [9]. This results in a linear time-periodic (LTP) system whose state-space matrix coefficients are $T_{g^{-}}$ periodic:

$$
\begin{aligned}
\frac{d \boldsymbol{x}(t)}{d t} & =\boldsymbol{A}(t) \boldsymbol{x}(t)+\boldsymbol{B}(t) \boldsymbol{u}(t), \\
\boldsymbol{y}(t) & =\boldsymbol{C}(t) \boldsymbol{x}(t)+\boldsymbol{D}(t) \boldsymbol{u}(t) .
\end{aligned}
$$

2) Signal representation: Both signals and periodic statespace coefficients are replaced by their Fourier series expansions. The complex-valued Fourier coefficients of the signals are time-varying whereas those of the state-space coefficients do not depend on time if $\omega_{g}=2 \pi / T_{g}$ :

$$
\begin{array}{cc}
\boldsymbol{x}(t)=\sum_{k=-\infty}^{+\infty} \boldsymbol{X}_{k}(t) e^{j k \omega_{g} t}, & \begin{array}{r}
\text { similarly } \\
\text { for } \boldsymbol{u}, \boldsymbol{y}
\end{array} \\
\boldsymbol{A}(t)=\sum_{k=-\infty}^{+\infty} \boldsymbol{A}_{k} e^{j k \omega_{g} t}, & \text { similarly } \\
\text { for } \boldsymbol{B}, \boldsymbol{C}, \boldsymbol{D}
\end{array}
$$

Equivalently, the signals can be expressed as exponentiallymodulated periodic signals, which results in a formulation directly in the Laplace domain [9].

Two types of operations must be considered: the time derivative of a signal, as in (10a), and the product of a signal and a periodic coefficient, as in (10b):

$$
\begin{aligned}
\frac{d \boldsymbol{x}(t)}{d t} & =\frac{d}{d t} \sum_{k=-\infty}^{+\infty} \boldsymbol{X}_{k}(t) e^{j k \omega_{g} t} \\
& =\sum_{k=-\infty}^{+\infty}\left(\frac{d \boldsymbol{X}_{k}(t)}{d t}+j k \omega_{g} \boldsymbol{X}_{k}(t)\right) e^{j k \omega_{g} t}, \\
\boldsymbol{A}(t) \boldsymbol{x}(t) & =\left(\sum_{k=-\infty}^{+\infty} \boldsymbol{A}_{k} e^{j k \omega_{g} t}\right)\left(\sum_{k=-\infty}^{+\infty} \boldsymbol{X}_{k}(t) e^{j k \omega_{g} t}\right) \\
& =\sum_{k=-\infty}^{+\infty}\left(\sum_{h=-\infty}^{+\infty} \boldsymbol{A}_{k-h} \boldsymbol{X}_{h}(t)\right) e^{j k \omega_{g} t} .
\end{aligned}
$$

Similar relations are obtained for the remaining terms in (8).

3) Harmonic balance: As for DPs, applying harmonic balance consists in equating same-frequency phasors in (10), which gives $\forall k$ :

$$
\begin{gathered}
\frac{d \boldsymbol{X}_{k}(t)}{d t}+j k \omega_{g} \boldsymbol{X}_{k}(t)=\sum_{h=-\infty}^{+\infty}\left(\boldsymbol{A}_{k-h} \boldsymbol{X}_{h}(t)+\boldsymbol{B}_{k-h} \boldsymbol{U}_{h}(t)\right) \\
\boldsymbol{Y}_{k}(t)=\sum_{h=-\infty}^{+\infty}\left(\boldsymbol{C}_{k-h} \boldsymbol{X}_{h}(t)+\boldsymbol{D}_{k-h} \boldsymbol{U}_{h}(t)\right)
\end{gathered}
$$

Equations (11) are a harmonic-domain LTI formulation equivalent to the LTP system of (8). A compact matrix representation is obtained using the following definitions:

$$
\begin{aligned}
& \mathcal{X}(t) \triangleq\left[\begin{array}{lllll}
\cdots & \boldsymbol{X}_{-1}^{T}(t) & \boldsymbol{X}_{0}^{T}(t) & \boldsymbol{X}_{+1}^{T}(t) & \cdots
\end{array}\right]^{T}, \begin{array}{r}
\text { similarly } \\
\text { for } \mathcal{U}, \mathcal{Y}
\end{array}
\end{aligned}
$$

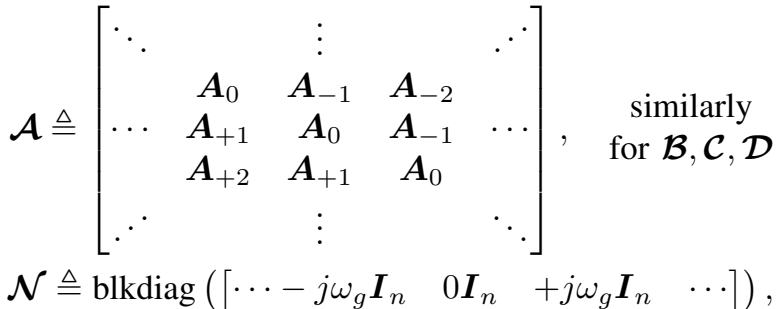

where $\boldsymbol{I}_{n}$ is the identity matrix of size $n$, i.e. the number of states in the LTP system. The HSS representation eventually reads as follows:

$$
\begin{aligned}
\frac{d \mathcal{X}(t)}{d t} & =(\mathcal{A}-\mathcal{N}) \mathcal{X}(t)+\mathcal{B U}(t) \\
\mathcal{Y}(t) & =\mathcal{C} \mathcal{X}(t)+\mathcal{D} \mathcal{U}(t)
\end{aligned}
$$

In this formulation, the signals appear as infinite vectors of complex-valued time-varying Fourier coefficients and the matrices $\mathcal{A}, \mathcal{B}, \mathcal{C}$ and $\mathcal{D}$ take the form of infinite blockToeplitz block-Hermitian matrices. The resulting state matrix used for stability assessment based on eigenvalues is $\mathcal{A}-\mathcal{N}$. The harmonic transfer function is obtained as:

$$
\mathcal{Y}(s)=\left(\mathcal{C}(s \boldsymbol{I}-\mathcal{A}+\mathcal{N})^{-1} \mathcal{B}+\mathcal{D}\right) \mathcal{U}(s) .
$$

\section{Truncated formulations}

The derivation of DPs and HSS is so far carried out for an infinite number of harmonic indices. In practice, only a finite set of harmonics is included so that the models are suitable for numerical calculations.

Two parameters are defined: $h_{m}$, the maximum index of the harmonic components necessary to exactly describe the steadystate periodic trajectory of the system, and $h_{t}$, the maximum index of the harmonic components included in the harmonic models. A priori, $h_{m}$ and $h_{t}$ are independent. Naturally, $h_{m}$ will be smaller for periodic trajectories described exactly by a truncated Fourier series than for trajectories described by a slowly converging Fourier series [17].

In truncated HSS models, the maximum harmonic index is limited to $h_{t}$, and all $n_{h}=2 h_{t}+1$ harmonic components between $-h_{t}$ and $+h_{t}$ are included in the formulation. In truncated DPs models, the maximum harmonic index is also limited to $h_{t}$, but not all harmonic components between $-h_{t}$ and $+h_{t}$ are usually included in the formulation. Depending 
on the harmonic content of the system steady-state periodic trajectory and the desired level of accuracy, a selection of DPs with specific harmonic indices is included in the model.

The simplest choice for harmonic-free $\mathrm{AC}$ systems is to include only fundamental-frequency (index-1) DPs for AC variables. For DC systems, only index-0 DPs are included. More elaborate selections of DPs indices may be considered when the steady-state periodic trajectory presents other harmonic components. For instance, index-0 DPs may be included for $\mathrm{AC}$ variables presenting a steady-state $\mathrm{DC}$ component. Index-1 DPs may be included for DC variables containing steady-state fundamental-frequency oscillations. Similarly, index- $k$ DPs can be included for variables presenting steadystate $k^{t h}$ harmonic oscillations. The selection of indices is further discussed in Sections IV and V.

\section{Theoretical comparison}

From the derivations of the two methods, it appears that the linearized set of DPs state-space equations relates to a harmonic state-space formulation, and that the HSS approach makes use of signals that relate to dynamic phasors, i.e. complex-valued time-varying Fourier coefficients. There is no separation of real and imaginary parts of the variables in HSS but both positive and negative frequencies are included, to the exception of the DC coefficient. In DPs models, the negativefrequency DPs are accounted for by means of complex conjugates, as expressed in (5). Therefore, a given harmonic index is represented by the same number of states in both methods.

Additionally, the real-valued DPs formulation results in a real-valued dynamic matrix while that of HSS is complexvalued.

As they originate from similar modeling hypotheses and their derivations follow the same steps (although in a different order), the infinite formulations of the two methods are equivalent in the sense that state-space models with same dynamics are obtained, although one method has real states (DP) whereas the other one has complex states (HSS). The equivalence of truncated formulations depends on the truncation orders and on which harmonic components are included in the DP model below the truncation order $h_{t}$, as demonstrated in Section V.

\section{Dynamic Equations of a Two-Level Voltage SOURCE CONVERTER}

This section describes the topology and control of a twolevel VSC, depicted in Fig. 1 and with parameters from Table II. The system is purposely kept very simple to emphasize the modeling approach in the following section. However, both DPs and HSS may be applied to more complex networks involving multiple converters for the study of control interactions. A VSC converter is studied instead of an LCC converter, although DPs and HSS methods can be applied to average models of LCC converters as well, for instance by using the switching function representation [18].

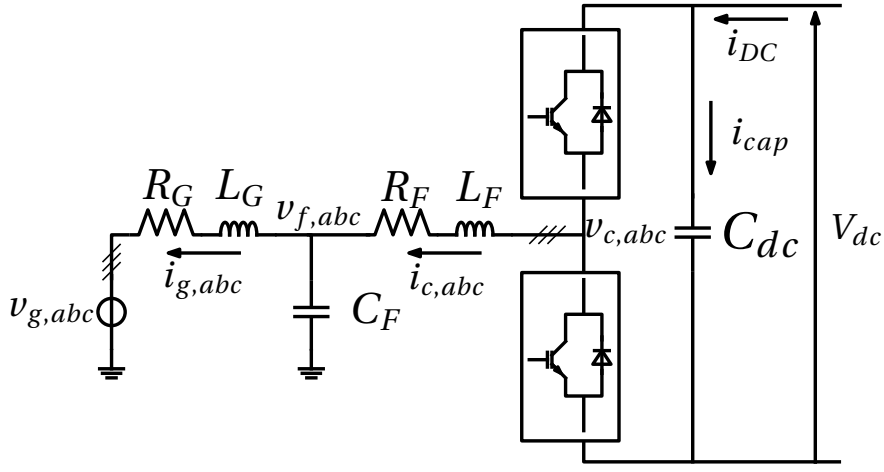

Fig. 1. Circuit diagram of the two-level VSC.

\section{A. Stationary-frame model}

An averaged model of the converter is considered, which implies that the terminal voltage of the converter for an arbitrary phase $\phi$ is given by:

$$
v_{c \phi}=m_{\phi} \frac{v_{d c}}{2},
$$

where $m_{\phi} \in[-1,1]$ is the modulation index of phase $\phi$, calculated by the controller. The per-phase operation of the converter is then modeled by means of four state variables: the AC-side current $i_{g}$, converter current $i_{c}$, filter voltage $v_{f}$ and DC voltage $v_{d c}$. The corresponding differential equations describing the converter dynamics for an arbitrary phase $\phi$ are:

$$
\begin{aligned}
L_{G} \frac{d i_{g \phi}}{d t} & =v_{f \phi}-v_{g \phi}-R_{G} i_{g \phi}, \\
L_{F} \frac{d i_{c \phi}}{d t} & =v_{c \phi}-v_{f \phi}-R_{F} i_{c \phi}, \\
C_{F} \frac{d v_{f \phi}}{d t} & =i_{c \phi}-i_{g \phi}, \\
C_{d c} \frac{d v_{d c}}{d t} & =i_{c a p} .
\end{aligned}
$$

In addition to the differential equations in (18), the power balance between the AC and DC sides is expressed in order to write the capacitor current in terms of the state variables. The power balance is written as:

$$
\begin{aligned}
v_{d c}\left(i_{d c}-i_{c a p}\right) & =v_{c a} i_{c a}+v_{c b} i_{c b}+v_{c c} i_{c c}, \\
\text { Leading to: } \quad i_{c a p} & =i_{d c}-\frac{1}{2}\left(m_{a} i_{c a}+m_{b} i_{c b}+m_{c} i_{c c}\right) .
\end{aligned}
$$

A further step is taken to define the dynamic model of the converter current controller, and integrate it into (18). As the model is derived in the stationary $a b c$ frame, per-phase fundamental-frequency resonant controllers are chosen for the control of the converter currents $i_{c \phi}$. The controller block diagram is given in Fig. 2. The equivalent state-space model of the second-order resonant controller is derived in terms of two additional states per phase, $\eta_{\phi}$ and $\gamma_{\phi}$, as:

$$
\begin{aligned}
\frac{d \eta_{\phi}}{d t} & =\gamma_{\phi}+K_{r}\left(i_{c \phi}^{*}-i_{c \phi}\right), \\
\frac{d \gamma_{\phi}}{d t} & =-\omega_{g}^{2} \eta_{\phi}, \\
m_{\phi} & =2\left(\eta_{\phi}+K_{p}\left(i_{c \phi}^{*}-i_{c \phi}\right)\right) .
\end{aligned}
$$


The sets of first-order differential equations in (18) and (20),

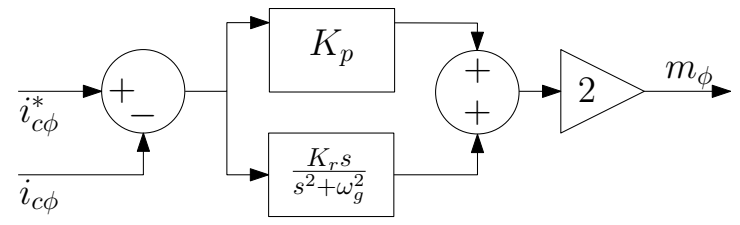

Fig. 2. Per-phase proportional-resonant controller.

together with the expressions of $v_{c \phi}, i_{c a p}$ and $m_{\phi}$ in (17), (19b) and (20c) respectively, constitute the complete dynamic model of the VSC and its controller. Its inputs are the AC voltages $v_{g, a b c}$, the DC current $i_{d c}$ and the current references $i_{c, a b c}^{*}$. Its 16 states are gathered in vector $\boldsymbol{x}$ :

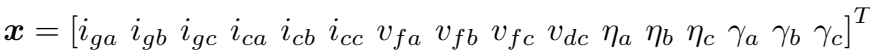

Because the insertion indices $m_{\phi}$ depend on states, products of states appear in the expressions of $v_{c \phi}$ and $i_{c a p}$. Consequently, the dynamic model is nonlinear. To the exception of $v_{d c}$, all states present a fundamental-frequency oscillation in steady state, making the system time-periodic. Specifically, the system steady-state periodic trajectory can be fully characterised by harmonic components up to $h_{m}=1$.

\section{B. Synchronous-frame equivalent model}

The nonlinear model developed in the previous paragraphs is formulated in the stationary $a b c$ frame. An equivalent $d q 0$ model expressed in the synchronous frame is obtained by applying the power-variant Park transformation to the set of differential equations. The resulting model is as follows:

$$
\begin{aligned}
& L_{F}\left(\frac{d i_{c d}}{d t}-\omega_{g} i_{c q}\right)=m_{d} \frac{v_{d c}}{2}-v_{f d}-R_{F} i_{c d} \\
& L_{F}\left(\frac{d i_{c q}}{d t}+\omega_{g} i_{c d}\right)=m_{q} \frac{v_{d c}}{2}-v_{f q}-R_{F} i_{c q} \\
& L_{F} \frac{d i_{c 0}}{d t}=m_{0} \frac{v_{d c}}{2}-v_{f 0}-R_{F} i_{c 0} \\
& C_{D C} \frac{d v_{d c}}{d t}=i_{d c}-\frac{3}{4}\left(m_{d} i_{c d}+m_{q} i_{c q}\right)-\frac{3}{2} m_{0} i_{c 0} \\
& \frac{d \eta_{d}}{d t}-\omega_{g} \eta_{q}=\gamma_{d}+K_{r}\left(i_{c d}^{\star}-i_{c d}\right) \\
& \frac{d \eta_{q}}{d t}+\omega_{g} \eta_{d}=\gamma_{q}+K_{r}\left(i_{c q}^{\star}-i_{c q}\right) \\
& \frac{d \eta_{0}}{d t}=\gamma_{0}+K_{r}\left(i_{c 0}^{\star}-i_{c 0}\right)
\end{aligned}
$$

where the equations relative to the states $i_{g \phi}, v_{f \phi}$ and $\gamma_{\phi}$ are obtained in the same manner but are not provided for conciseness. All variables of this $d q 0$ model have constant values in steady state. Therefore, its small-signal linear approximation is an LTI system.

\section{Application of the Modeling Methods}

In this section, the modeling principles of DPs and HSS are applied to the dynamic equations of the VSC.

\section{A. Dynamic phasors model}

The states and inputs in Equations (17) to (20) are replaced with their index- $k$ DPs and (4) is applied. This results in the generic index- $k$ set of DPs nonlinear time-invariant equations describing the VSC:

$$
\begin{aligned}
\frac{d\left\langle i_{g \phi}\right\rangle_{k}}{d t} & =\frac{\left\langle v_{f \phi}\right\rangle_{k}-\left\langle v_{g \phi}\right\rangle_{k}-R_{G}\left\langle i_{g \phi}\right\rangle_{k}}{L_{G}}-j k \omega_{g}\left\langle i_{g \phi}\right\rangle_{k} \\
\frac{d\left\langle i_{c \phi}\right\rangle_{k}}{d t} & =\frac{\left\langle v_{c \phi}\right\rangle_{k}-\left\langle v_{f \phi}\right\rangle_{k}-R_{F}\left\langle i_{c \phi}\right\rangle_{k}}{L_{F}}-j k \omega_{g}\left\langle i_{c \phi}\right\rangle_{k} \\
\frac{d\left\langle v_{f \phi}\right\rangle_{k}}{d t} & =\frac{\left\langle i_{c \phi}\right\rangle_{k}-\left\langle i_{g \phi}\right\rangle_{k}}{C_{F}}-j \omega_{g}\left\langle v_{f \phi}\right\rangle_{k} \\
\frac{d\left\langle v_{d c}\right\rangle_{k}}{d t} & =\frac{\left\langle i_{c a p}\right\rangle_{k}}{C_{d c}}-j k \omega_{g}\left\langle v_{d c}\right\rangle_{k} \\
\frac{d\left\langle\eta_{\phi}\right\rangle_{k}}{d t} & =\left\langle\gamma_{\phi}\right\rangle_{k}+K_{r}\left(\left\langle i_{c \phi}^{*}\right\rangle_{k}-\left\langle i_{c \phi}\right\rangle_{k}\right)-j k \omega_{g}\left\langle\eta_{\phi}\right\rangle_{k} \\
\frac{d\left\langle\gamma_{\phi}\right\rangle_{k}}{d t} & =-w_{g}^{2}\left\langle\eta_{\phi}\right\rangle_{k}-j k \omega_{g}\left\langle\gamma_{\phi}\right\rangle_{k} \\
\left\langle m_{\phi}\right\rangle_{k} & =2\left(\left\langle\eta_{\phi}\right\rangle_{k}+K_{p}\left(\left\langle i_{c \phi}^{*}\right\rangle_{k}-\left\langle i_{c \phi}\right\rangle_{k}\right)\right) \\
\left\langle i_{c a p}\right\rangle_{k} & =\left\langle i_{d c}\right\rangle_{k}-\sum_{\phi \in\{a, b, c\}} \sum_{l=-\infty}^{+\infty} \frac{\left\langle m_{\phi}\right\rangle_{l}\left\langle i_{c \phi}\right\rangle_{k-l}}{2}
\end{aligned}
$$

With the harmonic content of the input variables defined, the steady-state operating point is either obtained through timedomain integration of the nonlinear dynamic equations, or by equating the time derivatives in (23) to zero and solving for the states. Finally, the model is linearized around this operating point.

\section{B. Harmonic state-space model}

In HSS, the nonlinear system is first linearized around a steady-state periodic trajectory. The resulting small-signal linear system is:

$$
\begin{aligned}
L_{G} \frac{\widetilde{d i}_{g \phi}}{d t} & =\widetilde{v}_{f \phi}-\widetilde{v}_{g \phi}-R_{G} \widetilde{i}_{g \phi} \\
L_{F} \frac{\widetilde{i}_{c \phi}}{d t} & =\frac{1}{2}\left(\bar{m}_{j} \widetilde{v}_{d c}+\widetilde{m}_{j} \bar{v}_{d c}\right)-\widetilde{v}_{f \phi}-R_{F} \widetilde{i}_{c \phi} \\
C_{F} \frac{d \widetilde{v}_{f \phi}}{d t} & =\widetilde{i}_{c \phi}-\widetilde{i}_{g \phi} \\
C_{d c} \frac{d \widetilde{v}_{d c}}{d t} & =\widetilde{i}_{d c}-\frac{1}{2} \sum_{\phi=a b c}\left(\bar{m}_{\phi} \widetilde{i}_{c \phi}+\widetilde{m}_{\phi} \bar{i}_{c \phi}\right) \\
\frac{d \widetilde{\eta}_{\phi}}{d t} & \left.=\widetilde{\gamma}_{j}+K_{r} \widetilde{i}_{c \phi}^{*}-\widetilde{i}_{c \phi}\right) \\
\frac{d \widetilde{\gamma}_{\phi}}{d t} & =-\omega_{g}^{2} \widetilde{\eta}_{j}
\end{aligned}
$$

where bar ${ }^{*}$ and tilde $\sim$ notations refer to periodic coefficients and small-signal perturbations, respectively. As for DPs, the Fourier coefficients of the periodic quantities in steady state can either be obtained through time-domain integration or from the resolution of the nonlinear system in the harmonic domain with the time-derivatives set equal to zero. Finally, (24) is brought to the HSS matrix form as in (15). 


\section{Models used for comparison}

Keeping in mind that $h_{m}=1$ for this study case, it is of interest to compare the models with different values of $h_{t}$ and different sets of DPs indices. For this reason, the next section considers several versions of the DP and HSS models:

- Model DP1a contains index-1 DPs for $v_{g \phi}, i_{g \phi}, i_{c \phi}, v_{f \phi}$, $\eta_{\phi}$ and $\gamma_{\phi}$, and index-0 DPs for $v_{d c}$ and $i_{d c}$.

- Model DP1b is an extension of DP1a, including index-0 DPs for the AC variables, and index-1 DPs for the DC variables.

- Model DP3 is an extension of DP1b, including all DPs up to $k=3$.

- The HSS models have truncation indices $h_{t}$ of $0,1,3$ and 16 respectively. In particular, HSSO is only applicable to LTI systems as it can only describe systems with a constant steady-state operating point.

A summary of the models is given in Table I, where $\mathrm{N}$ refers to the resulting number of states in the frequency-lifted models.

TABLE I

SUMMARY OF MODEL DESIGN CHOICES

\begin{tabular}{|c|c|c||c|c|c|c|}
\hline \multicolumn{3}{|c||}{ Dynamic phasors } & \multicolumn{4}{c|}{ Harmonic state-space } \\
\hline Name & DP indices per state & $\mathbf{N}$ & Name & $\boldsymbol{h}_{t}$ & $\boldsymbol{n}_{\boldsymbol{h}}$ & $\mathbf{N}$ \\
\hline DP1a & AC: $k=1$, DC: $k=0$ & 31 & HSS0 & 0 & 1 & 16 \\
\hline DP1b & all: $k=0,1$ & 48 & HSS1 & 1 & 3 & 48 \\
\hline DP3 & all: $k=0,1,2,3$ & 112 & HSS3 & 3 & 7 & 112 \\
\hline \multicolumn{2}{|l}{} & HSS16 & 16 & 33 & 528 \\
\hline
\end{tabular}

TABLE II

CONVERTER DESIGN DATA

\begin{tabular}{|c|c|c|c|}
\hline Parameter & Description & Value & Unit \\
\hline$R_{G}$ & Grid-side resistance & 0.012 & $\Omega$ \\
\hline$L_{G}$ & Grid-side inductance & 42.9 & $\mathrm{mH}$ \\
\hline$R_{F}$ & Filter resistance & 0.3429 & $\Omega$ \\
\hline$L_{F}$ & Filter inductance & 62.9 & $\mathrm{mH}$ \\
\hline$C_{F}$ & Filter capacitance & 2.3463 & $\mu F$ \\
\hline$C_{d c}$ & DC-side capacitance & 62.7 & $\mu F$ \\
\hline$v_{d c}$ & Rated DC voltage & 620.54 & $\mathrm{kV}$ \\
\hline$v_{g, L L}^{R M S}$ & RMS value of the AC grid line voltage & 380 & $\mathrm{kV}$ \\
\hline$K_{P}$ & Current controller proportional gain, normalized with $v_{d c}$ & $10^{-5}$ & $1 / A$ \\
\hline$K_{R}$ & Current controller resonant gain, normalized with $v_{d c}$ & 1 & $1 / A s$ \\
\hline$i_{c}^{R M S}$ & RMS value of the converter current controller reference & 1.5193 & $\mathrm{kA}$ \\
\hline
\end{tabular}

\section{EIGENVALUES AND TRANSFER FUNCTIONS ANALYSIS}

In this section, the eigenvalues and transfer functions of the models of Table I are analysed. The calculations of eigenvalues and transfer functions use MatLab's eig and freqresp functions respectively.

Fig. 3 compares the eigenvalues of models DP1a, DP1b and HSS1. For clarity, only the positive imaginary axis is displayed, the complex conjugates of the eigenvalues being obtained by symmetry. It is observed that the eigenvalues of models DP1b and HSS1 are strictly equivalent, whilst some eigenvalues do not appear in model DP1a, which naturally presents a smaller number of states. All the models display some overlapping eigenvalues, the number of which is indicated with a dashed arrow. The eigenvalues whose real part is close to -1 are not discussed for brevity. It is well known

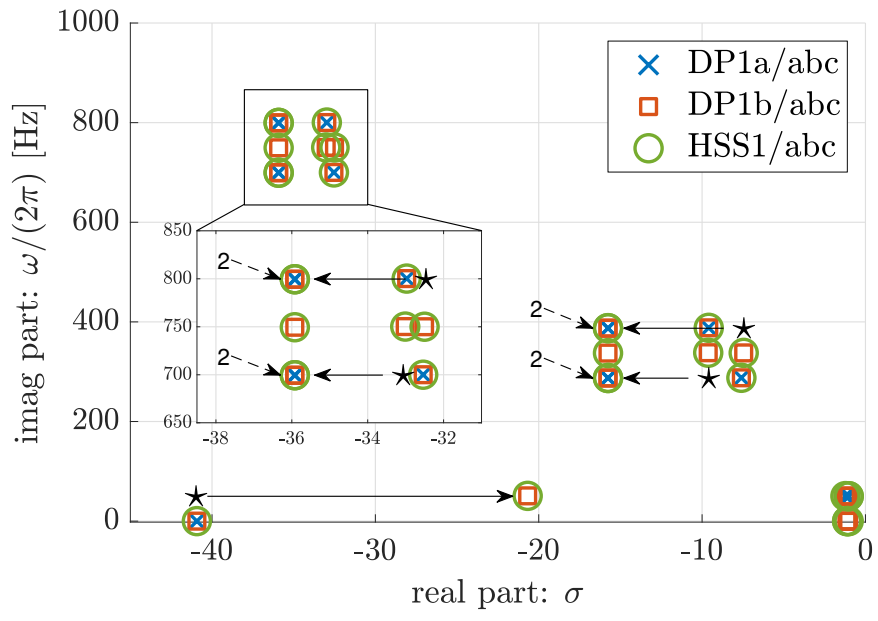

Fig. 3. Eigenvalues of DP1a, DP1b and HSS1 models. Only the positive $\mathrm{y}$-axis is provided for clarity.

that the eigenvalues of non-truncated HSS models appear as infinite sets of duplicated eigenvalues translated along the imaginary axis by multiples of the fundamental frequency [9], which results in infinite vertical lines in the complex plane. When the HSS state matrix is truncated, the eigenvalues form vertical lines of finite length, and some may present a deviation near the ends of the lines [12], to the exception of LTI systems. As explained later, not all vertical lines present such shifted eigenvalues. These eigenvalues are correct eigenvalues of the truncated state matrix, but the truncated formulation is, as such, not an intact representation of the LTP system. Consequently, the shifted eigenvalues are spurious and should be disregarded. In Fig. 3, stars ( $\star$ ) give the normally expected location of the spurious eigenvalues as part of the vertical lines, and solid arrows indicate their shift towards their eventual location as a result of the model truncation. This leads in some cases to the aforementioned overlapping eigenvalues, only one of which is spurious.

Fig. 4 presents the eigenvalues of the DP3 and HSS3 models. It is again apparent that their eigenvalues match perfectly. As the steady-state periodic trajectory does not contain harmonics beyond the fundamental frequency $\left(h_{m}=1\right)$, the additional Fourier coefficients $\boldsymbol{A}_{ \pm 2, \pm 3}$ that result from the increased truncation order $\left(h_{t}=3\right)$ are null. As a result, the vertical lines characteristics of HSS are longer but their real parts remain the same. The spurious behaviour still affects the eigenvalues close to the end of the lines.

In Fig. 5, the eigenvalues of the $a b c$-frame model (HSS3/abc) are compared with those of the $d q 0$-frame model (HSS0/dq0 and HSS3/dq0). Being time-invariant, the synchronous-frame model does not require frequency lifting and can be accurately described with the HSSO model. Consequently, the $\mathrm{HSS} 3 / \mathrm{dq} 0$ model duplicates and translates the eigenvalues of the HSS0/dq0 model along the imaginary axis and does not introduce any spurious eigenvalues. The real parts of the $d q 0$ eigenvalues match those of the vertical lines of 


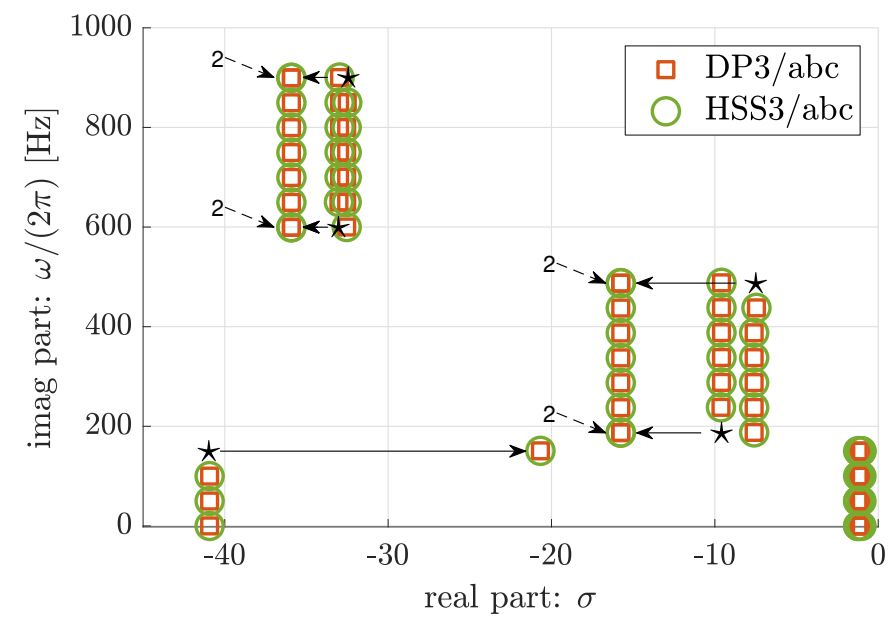

Fig. 4. Eigenvalues of DP3 and HSS3 models. Only the positive y-axis is provided for clarity.

HSS3/abc. The application of Park's transformation introduces a $\pm j \omega_{g}$ (imaginary part) shift of the eigenvalues related to $d$ and $q$ variables with respect to those of the $a b c$ model (e.g. vertical lines with real parts equal to -9.59 and -7.59 ), while the eigenvalues related to the zero sequence do not present such shift (e.g. vertical line with real part equal to -15.76). Unlike the real-part shift of spurious eigenvalues, this imaginary-part shift is a natural consequence of Park's transformation. This classification per sequence also shows that, in the present case, only the vertical lines related to positive and negative sequences in the stationary-frame models yield spurious eigenvalues while those related to the zerosequence do not.

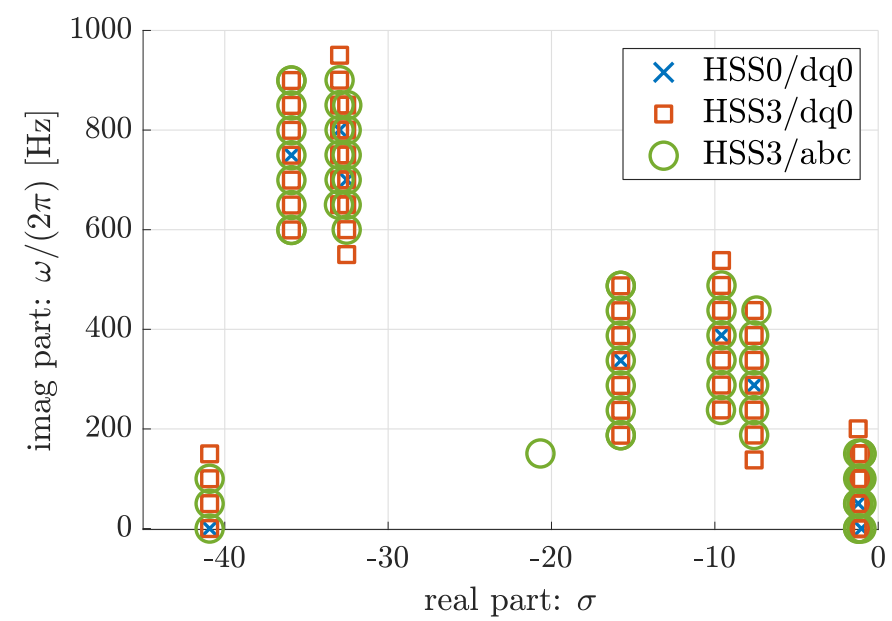

Fig. 5. Eigenvalues of HSSO and HSS3 applied to the dq0 system, compared with HSS3 applied to the abc system. Only the positive y-axis is provided.

The previous analyses raise the question of choosing an appropriate truncation order. In [17], it is suggested that $h_{t}$ is large enough when the amount and position of eigenvalues within the fundamental frequency strip ${ }^{3}$ of the complex plane have converged (i.e. when all vertical lines have a non-spurious eigenvalue in that strip). This situation is illustrated in Fig. 6 with model HSS16/abc. However, such an increased truncation order is only necessary when the stability assessment is performed with the Generalised Nyquist Criterion limited to the fundamental frequency strip, as described in [17]. If the stability assessment is based on the horizontal position of the vertical lines, defined as the real part of their centermost eigenvalue, it is sufficient to have $h_{t} \geq h_{m}$. In particular, selecting $h_{t}>h_{m}$ increases the number of eigenvalues in the vertical lines, but does not affect their horizontal position. Moreover, it is obvious that no accurate stability assessment can be performed if $h_{t}<h_{m}$, unless all steady-state harmonics of index $k \in\left\{h_{t}+1, \ldots, h_{m}\right\}$ are negligible. In this specific case study, $h_{m}=1$, so $h_{t} \geq 1$ is recommended.

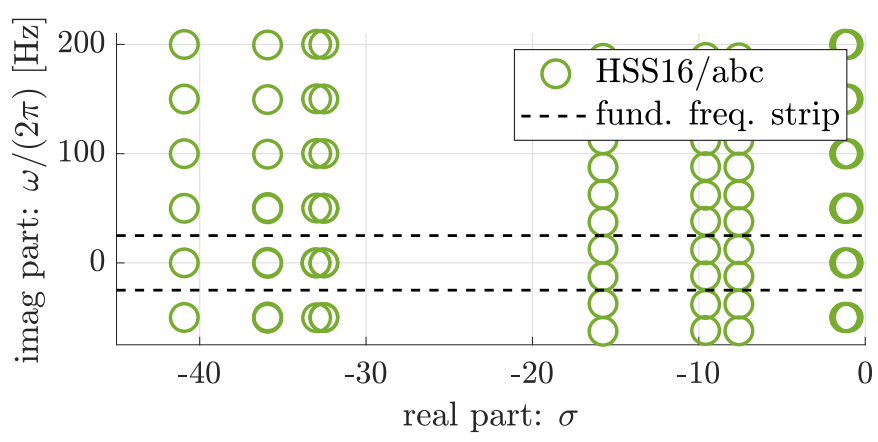

Fig. 6. Eigenvalues of model HSS16. Only part of the y-axis is displayed for clarity. Not all eigenvalues are visible.

The transfer functions are calculated using (7) and (16) for the DP and HSS systems respectively. Fig. 7 provides the DC-side impedance $v_{d c}(j \omega) / i_{d c}(j \omega)$ of the $a b c$ frame model. In both DP1a and HSS1, this transfer function describes the dynamics from the DC current $i_{d c}$ to the DC voltage $v_{d c}$, without frequency shift between input and output. It is observed that the two methods deliver the same transfer function.

When the phasors of the DP model are split between real and imaginary parts, it is not straightforward to retrieve the AC-side admittance $i_{g \phi}(j \omega) / v_{g \phi}(j \omega)$ under the form of a single transfer function, as it takes the form of a matrix transfer function from $\left\langle v_{g \phi}\right\rangle_{1}^{R}(j \omega)$ and $\left\langle v_{g \phi}\right\rangle_{1}^{I}(j \omega)$ to $\left\langle i_{g \phi}\right\rangle_{1}^{R}(j \omega)$ and $\left\langle i_{g \phi}\right\rangle_{1}^{I}(j \omega)$. However, if the extended model DP1b is used, then the transfer function from $v_{g \phi}(j \omega)$ to $i_{g \phi}(j \omega)$ (without frequency shift) can be obtained as $\left\langle i_{g \phi}\right\rangle_{0}(j \omega) /\left\langle v_{g \phi}\right\rangle_{0}(j \omega)$. This last transfer function is compared with the corresponding one of HSS in Fig. 8, where their equivalence is again clear. Unlike the real-valued DP models, the complex-valued HSS models provide all transfer functions describing the harmonic couplings within the LTP system, i.e. describing the dynamics both with and without frequency shifts between input and output variables.

\footnotetext{
${ }^{3}$ The fundamental frequency strip contains all complex numbers $\lambda$ such that $-j \omega_{g} / 2+j \xi<j \operatorname{Im}(\lambda) \leq j \omega_{g} / 2+j \xi$, with $\omega_{g}$ the fundamental angular frequency and $\xi$ generally equal to zero.
} 

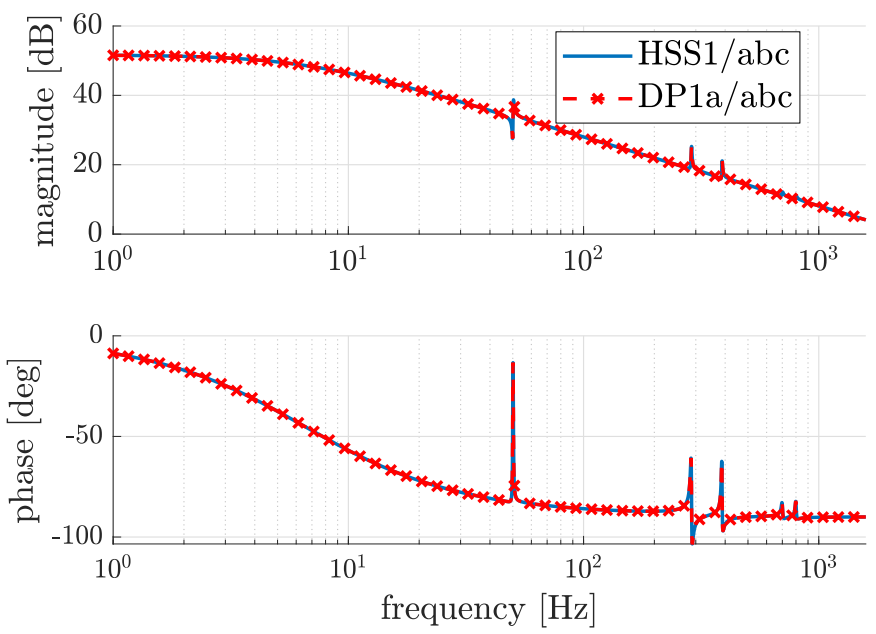

Fig. 7. DC-side impedance: transfer function from $i_{d c}^{0}$ to $v_{d c}^{0}$.
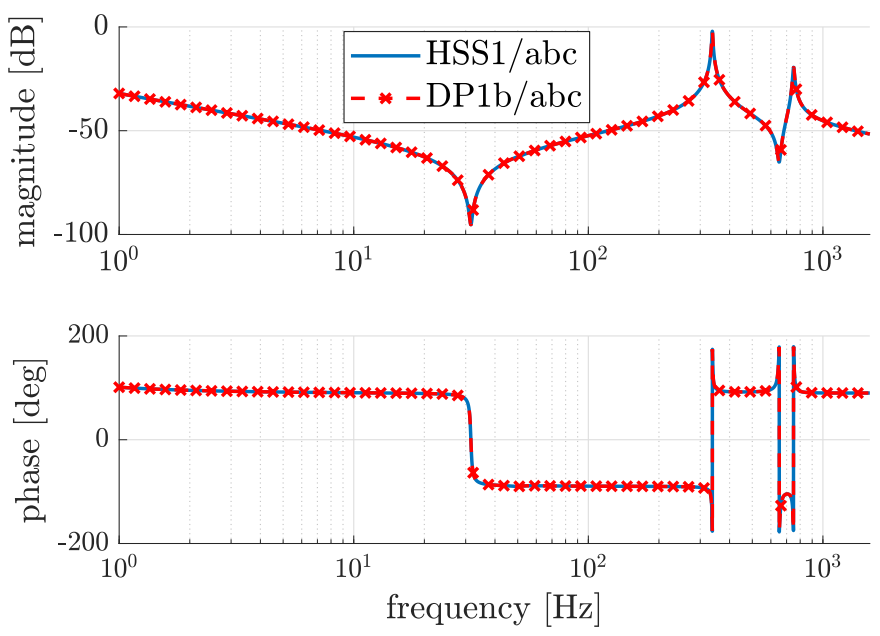

Fig. 8. AC-side admittance: transfer function from $v_{g a}^{0}$ to $i_{g a}^{0}$.

\section{COMPARISON OF AdVANTAGES AND SHORTCOMINGS}

The analyses of eigenvalues and transfer functions in the previous section show that the presented modeling methods share many characteristics. The DP method results in a realvalued model of the system, whereas the HSS method develops a complex-valued model. Regarding eigenvalues analysis, even though the system matrices are different, both methods give the same eigenvalues when the extended DP formulation is applied. Truncated models obtained using these methods contain spurious eigenvalues. When the DP method is applied with a simplified harmonic content (as for DP1a), a lower-order system representation can be obtained. Concerning frequencydomain analysis, it is worth mentioning that the real-valued DP method presents limitations regarding AC-side admittance calculations, as it is not straightforward to define a unified admittance representation. Thus, the real-valued DP method is more suitable for eigenvalue-based stability analysis than for impedance-based stability analysis.

\section{CONCLUSIONS}

This paper presented an in-depth comparison of two promising methods to model power-electronic converters for smallsignal stability analysis: dynamic phasors and harmonic state space. Their derivations were introduced and a theoretical comparison was carried out to highlight their similarities and differences. Both methods were applied to a two-level VSC, after which the systems eigenvalues and transfer functions were compared.

The two methods are obtained from similar hypotheses and their derivations follow the same steps, leading to statespace models with same dynamic behaviour when same design choices are used. Using real-valued variables in the DPs models results in complexities when impedance-based stability analysis is of interest.

As a result of truncation, all frequency-lifted models of LTP systems present spurious eigenvalues that should be disregarded for the stability assessment. These spurious eigenvalues translate the fact that truncated formulations are not intact representations of the LTP systems. In the chosen study case, only positive and negative sequences were subject to spurious eigenvalues due to truncation.

Lastly, a distinction was made between maximum harmonic index $h_{m}$ of periodic trajectories and truncation order $h_{t}$ of models, suggesting that accurate eigenvalue-based stability assessment is possible when $h_{t} \geq h_{m}$. [19]

\section{REFERENCES}

[1] X. Wang and F. Blaabjerg, "Harmonic stability in power electronic-based power systems: Concept, modeling, and analysis," IEEE Trans. on Smart Grid, vol. 10, no. 3, pp. 2858-2870, May 2019.

[2] X. Yue, X. Wang, and F. Blaabjerg, "Review of small-signal modeling methods including frequency-coupling dynamics of power converters," IEEE Trans. on Power Elec., vol. 34, no. 4, pp. 3313-3328, April 2019.

[3] S. R. Sanders et al., "Generalized averaging method for power conversion circuits," IEEE Trans. on Power Elec., vol. 6, no. 2, pp. 251-259, April 1991.

[4] P. Mattavelli, G. C. Verghese, and A. M. Stankovic, "Phasor dynamics of thyristor-controlled series capacitor systems," IEEE Trans. on Power Systems, vol. 12, no. 3, pp. 1259-1267, Aug 1997.

[5] A. M. Stankovic, B. C. Lesieutre, and T. Aydin, "Modeling and analysis of single-phase induction machines with dynamic phasors," IEEE Trans. on Power Systems, vol. 14, no. 1, pp. 9-14, Feb 1999.

[6] M. C. Chudasama and A. M. Kulkarni, "Dynamic phasor analysis of ssr mitigation schemes based on passive phase imbalance," IEEE Trans. on Power Systems, vol. 26, no. 3, pp. 1668-1676, Aug 2011.

[7] D. C. Patel and M. C. Chandorkar, "Small-signal transient analysis of induction machines with stator inter-turn faults using dynamic phasors," in Proc. 2012 IEEE Energy Conversion Congress and Exposition (ECCE), Sep. 2012, pp. 3008-3015.

[8] O. C. Sakinci and J. Beerten, "Generalized dynamic phasor modeling of the mmc for small-signal stability analysis," IEEE Trans. on Power Deliv., vol. 34, no. 3, pp. 991-1000, June 2019.

[9] N. M. Wereley, "Analysis and control of linear periodically time varying systems," Ph.D. dissertation, Department of Aeronautics and Astronautics, Massachusetts Institute of Technology (MIT), 1991.

[10] J. J. Rico, M. Madrigal, and E. Acha, "Dynamic harmonic evolution using the extended harmonic domain," IEEE Trans. on Power Deliv., vol. 18, no. 2, pp. 587-594, April 2003.

[11] J. S.-Ramirez et al., "Frequency domain methods for accuracy assessment of wideband models in electromagnetic transient stability studies," IEEE Trans. on Power Deliv., vol. 35, no. 1, pp. 71-83, Feb 2020.

[12] V. Salis et al., "Stability boundary analysis in single-phase gridconnected inverters with pll by ltp theory," IEEE Trans. on Power Elec., vol. 33, no. 5, pp. 4023-4036, May 2018. 
[13] J. Lyu et al., "Harmonic state-space based small-signal impedance modeling of a modular multilevel converter with consideration of internal harmonic dynamics," IEEE Trans. on Power Elec., vol. 34, no. 3, pp. 2134-2148, March 2019.

[14] N. Blin et al., "A comparison of harmonic modeling methods with application to control of switched systems with active filtering," in 2019 18th European Control Conference (ECC), June 2019, pp. 4198-4203.

[15] S. Bittanti and P. Colaneri, Periodic Systems: Filtering and Control, 1st ed. Springer-Verlag London, 2009.

[16] A. H. Nayfeh and D. T. Mook, Nonlinear Oscillations. John Wiley \& Sons, Ltd, 1995.

[17] N. M. Wereley and S. R. Hall, "Linear time periodic systems: Transfer function, poles, transmission zeroes and directional properties," in 1991 American Control Conference, June 1991, pp. 1179-1184.

[18] M. Daryabak et al., "Modeling of lcc-hvdc systems using dynamic phasors," IEEE Trans. on Power Deliv., vol. 29, no. 4, pp. 1989-1998, Aug 2014.

[19] P. De Rua, O. C. Sakinci, and J. Beerten, "Comparative Study of Dynamic Phasor and Harmonic State-Space Modeling for Small-Signal Stability Analysis," Electric Power Systems Research, vol. 189, no. 106626, 2020. 